\title{
COGNICIÓN, JUEGO Y APRENDIZAJE: UNA PROPUESTA PARA EL AULA DE LA PRIMERA INFANCIA
}

Cognition, play and learning: a proposal for the early childhood classroom Cognição, Jogo e Aprendizagem: uma proposta para aulas na primeira infância

María Francisca Muñoz Oyarce (1)

Alejandro Almonacid Fierro (2)

(1) Universidad Tecnológica de Chile. Fono: +56 71 510057. Correo electrónico: maria.munoz97@inacapmail.cl

(2) Universidad Autónoma de Chile.

\section{Resumen}

El artículo que a continuación se presenta pretende conectar tres dominios de alta significación para el aula escolar, vale decir, cognición, juego y aprendizaje, preferentemente desarrollados durante la primera infancia. Lo anterior, en el bien entendido que la noción de aprendizaje, desde la perspectiva de las neurociencias, ha puesto su foco en la comprensión de cómo el cerebro funciona, resaltando el papel de la memoria y fundamentalmente de la relación indisociable cuerpo-mente.

En la actualidad, y especialmente pensando en la educación del siglo XXI, resulta del todo pertinente la comprensión de la base biológica de la cognición humana, reconociendo que las manifestaciones comportamentales del sujeto son fruto de la interacción del individuo con el medio en que vive. Por otro lado, lo más relevante de las neurociencias hoy en día, es la formulación de directrices y orientaciones pedagógicas que busquen optimizar los procesos de enseñanza-aprendizaje en la escuela, a objeto de favorecer ejercicios cognitivos de mayor complejidad.

Finalmente, incorporar una perspectiva preferentemente lúdica en el aula de la primera infancia, es un desafío para los actuales y futuros educadores, toda vez que la escuela es siempre la continuación de la vida, y en la vida como bien lo sabemos, los niños aprenden jugando. En consecuencia, la intencionalidad educativa del juego se convierte en una premisa pedagógica-didáctica. 
Palabras Clave: educación, juego, cognición, aprendizaje.

\begin{abstract}
The article presented below, aims to connect three domains of high significance for the classroom, that is, cognition, play and learning, preferably developed during early childhood. This, on the understanding that the notion of learning from the perspective of neuroscience, has put its focus on understanding how the brain works, highlighting the role of memory and fundamentally inseparable from the mind-body relationship.

At present, especially considering education of the century, it is totally appropriate understanding of the biological basis of human cognition, recognizing that the behavioral manifestations of the subject are the result of the individual's interaction with the environment they live. On the other hand, the most relevant of neuroscience today is the development of guidelines and teaching guidelines that try to optimize the processes of teaching and learning in the school, in order to encourage more complex cognitive exercises.
\end{abstract}

Finally, preferably incorporating a playful perspective on early childhood classroom it is a challenge for current and future educators, since the school is always the continuation of life, and life as we all know, children learn playing. Consequently, the educational purpose of the game becomes a pedagogical-didactic premise.

Keywords: education, play, cognition, learning.

\title{
Resumo
}

$\mathrm{O}$ artigo que à continuação se apresenta, pretende conectar três domínios de alto significado para a dinâmica escolar, quer dizer, cognição, jogo e aprendizagem; preferencialmente desenvolvidos durante a primeira infância. Entendendo a noção de aprendizagem desde a perspectiva das neurociências, o foco é colocado na compreensão de como o cérebro funciona, ressaltando o papel da memória e, fundamentalmente, da relação indissociável corpo-mente.

Na atualidade, e especialmente pensando na educação do século XXI, resulta pertinente a compreensão da base biológica da cognição humana, reconhecendo que as manifestações 
comportamentais do sujeito são fruto da interação do indivíduo com o meio em que vive. Por outro lado, o mais relevante das neurociências, hoje em dia, é a formulação de diretrizes e orientações pedagógicas que busquem otimizar os processor de ensinoaprendizagem na escola, objetivando favorecer exercícios cognitivos de maior complexidade.

Finalmente, incorporar uma perspectiva preferencialmente lúdica nas aulas na primeira infância, é um desafio para os atuais e futuros educadores, toda vez que a escola é sempre a continuação da vida, e na vida, como bem sabemos, as crianças aprendem jogando (brincando). Em consequência, a intencionalidade educativa do jogo se converte em uma premissa pedagógica-didática.

Palavras Chave: educação, jogo, cognição, aprendizagem.

\section{Introducción}

Al intentar aproximarnos al fenómeno del juego, surge la necesaria reflexión hacia el interior del jardín o centro pre-escolar, y más específicamente al interior del aula, toda vez que es en este lugar donde debieran generarse las mayores condiciones para el aprendizaje. De hecho, la escuela es la institución social encargada de transmitir la cultura, de crearla y re-crearla, y de desarrollar progresivos procesos de socialización, en el marco de los valores y principios que la sociedad ha determinado. No obstante, la tarea preferente del centro educativo es generar instancias de aprendizaje con y para los estudiantes. En este sentido intentaremos analizar cómo el juego impacta y se hace cargo de aquellos procesos, en el bien entendido que el explosivo avance en las neurociencias de los últimos 30 años viene a confirmar lo relevante que resulta el juego para el desarrollo del aprendizaje de niños(as) en dimensiones tan diversas como los aspectos motores, cognitivos, afectivos y sociales.

Hoy, el juego es un lujo de pueblos rurales, en donde el barro, las piedras, los árboles y la lluvia son cómplices de la cotidianidad de de niños y niñas que crean y construyen historias entre paisajes de valles y montañas. Mientras, en la ciudad, el juego se desvanece bajo la mirada impávida de padres y educadores que parecieran temer a la 
creación y al movimiento. Se ha olvidado la importancia y la necesidad innata de explorar, de sentir, dejándola a cargo de imágenes insípidas cargadas de modernidad y quietud.

Animar a los niños a jugar y explorar les ayuda a aprender y desarrollarse social, emocional, física e intelectualmente. La importancia del juego en el desarrollo cognitivo no puede pasarse por alto. El juego es fundamental para el aprendizaje de los niños, sin importar el contenido real. El proceso del juego es una poderosa experiencia, el aprendizaje de múltiples facetas. Los juegos para niños involucran la exploración, la experimentación del lenguaje, la cognición y el desarrollo de habilidades sociales (UNICEF, 2012).

Entonces, ¿qué sucede con los espacios para crear y desarrollar el juego?, ¿sería posible jugar y aprender?, ¿podría ser el jardín infantil un lugar para jugar?

\section{Primer Momento: El Caos}

Gritos, risas y empujones, unos arriba, otros abajo, saltos, juguetes que se pasan, que se quitan, lloriqueos momentáneos, correr de un lado hacia otro, se pillan, se tocan, se ríen, y vuelven a reír, algunos se esconden, parece que desaparecen, otros los observan, se guiñan los ojos y vuelven al juego travieso, diverso, generoso, espontaneo..., todo aquello en medio de un salón, que podría ocuparse para diferentes actividades, pero este día de invierno, inmediatamente, se ha transforma en un patio de juegos.

Cuando los niños juegan se apropian del espacio y del tiempo, para habitarlo y transitarlo de una manera particular, su adaptabilidad y flexibilidad dan respuestas a necesidades, deseos y gustos, por ello la esencia misma del juego es siempre una expresión de la experiencia subjetiva de los niños (Medina, 2013).

La complicidad se hace presente permitiendo organizar diferentes grupos dispersados en el amplio espacio compartido, sin embargo, tres golpes de palmas indican el fin..., ¿del comienzo?

\section{Segundo Momento: El Orden}

Formados en fila "india" ingresan al aula, se sientan como absorbidos por una tierra pantanosa inmovilizando cualquier intención. 
Tal vez sea el invierno, que irrumpe y atemoriza la fascinación del movimiento expresado en su máxima pureza y libertad. Dejando inquietas las ideas, durmiendo la subjetividad.

No es extraño que alguno de nosotros piense que esté bien, que la escuela es un lugar para aprender. Otorgando al aprendizaje el papel antagónico del juego. Lo anterior se puede sustentar en aquellos planteamientos de la psicología conductista tan característicos de la primera parte del siglo XX, en donde el orden, la disciplina, el silencio, la estructura, la jerarquía, en fin, la clase tradicional y algo militarizada es lo que predomina, toda vez que se entiende que el conocimiento es información que en algún sentido se transmite y se deposita, cuestión que tan bien identificó Paulo Freire (2002) al categorizar a este tipo de educación como Bancaria.

Desde esta concepción, aprender mejor significa quietud y atención, es decir, niños y niñas están condenados a ser alumnos deficientes, ya que por naturaleza necesitan del movimiento y por ende del juego, conjugación en extinción en las aulas de hoy.

Quietos, muy quietos... "saquen su colación” es la nueva señal, no permito sorprenderme. Creemos, con esperanza, que todo cambiará. En algún momento nuestra formación constructivista se reflejará dejándonos impresionados y dichosos.

iiiSilencio!!! Es curiosa esta palabra, en ese instante se vuelve cruel y amenazadora, está lejos de lo que significa acoger, escuchar, empatizar. No obstante, es real, es una escuela particular, es un nivel pre-escolar y es el año 2014.

Vamos a trabajar con el libro de números. ¿Cuántos sapos hay en el lago?, ¿dónde está el 5?, márquenlo. ¡Muy bien!... Ahora vamos a contar...

Sin duda, niños y niñas cuentan sin dificultad de 1 a 10 ; de 10 a 20, pero ¿comprenden realmente lo que están realizando?, ¿tiene sentido para ellos? ¿podrían aprender lo mismo jugando con muñecas, autitos o legos?

\section{Tercer Momento: La Incertidumbre}

No hubo palmadas en las manos, no existió la fila india, nadie pidió "silencio", nadie dijo 'cállense', no hubo instrucciones, los rostros de los niños no reflejaron angustia, por el 
contrario, la maestra ingresó al aula, los niños y niñas se le suben a los brazos, otros aún tironean los juguetes, otros en un rincón pelean y ríen, los más corren y corren tocándose, y gritando. De pronto la profesora deja sus libros y en medio del 'desorden', pone una música que los invita a todos a bailar y a cantar, los niños y niñas se integran al nuevo juego de una manera natural, fluida, de a poco, dejan los juguetes, los pelos del compañero, las sillas y se incorporan al nuevo juego en el que todos participan. Posteriormente, la música va cambiando de intensidad y los niños y niñas comienzan a ejercitar sus brazos, sus piernas con movimientos suaves y ondulatorios, parecidos a una sesión de Tai Chi, luego se para la música y ya todos sentados alrededor de la maestra comparten los nuevos desafíos para la jornada a través de una conversación respetuosa, horizontal, en la que los niños y niñas participan, conversan, se ríen, de tal manera que todo ha fluido en un continuum en donde no se observan interrupciones, órdenes, retos, muy por el contrario, es la emergencia de una situación nueva, en que ha primado la armonía y las diversas sensibilidades del ser humano.

Recurrimos a Morin $(2001 ; 2005)$ para poder conectar las vivencias de una visita a una escuela durante los primeros meses del presente año escolar, toda vez que este autor, padre de la teoría de la complejidad, realiza una fuerte crítica al paradigma de la simplicidad, sustentado en una racionalidad Cartesiana que privilegia el orden y la organización, en el que el todo no es más que la suma de las partes, presentando un paradigma holístico, hologramático, sistémico y eminentemente complejo en el que el todo es más, mucho más, que la suma de las partes.

\section{Una primera aproximación a los estudios acerca del juego}

El juego es lenguaje primitivo y profundo, es la historia de los niños y niñas que inmersos en el mundo de los adultos escapan en una especie de catarsis liberadora y mágica. Dejando entrever dolores, heredables de lo cotidiano, de las experiencias, de lo más alegre, de lo más real, de sus vidas, que ellos inocentemente revelan al jugar. El juego es el rol familiar y social de un pueblo, de una ciudad, el juego trasluce lo más esencial, el color del alma sin editar.

Hoy, por diferentes razones y sin desmerecer la tecnología, los niños han dejado de jugar, pero no es sólo eso, padres y educadores avalan implícitamente esta muerte 
corporal y creativa, tanto individual como colectiva. Se quiebran los espacios y los tiempos de juego, dejando con ello escapar las etapas cruciales en favor de un desarrollo holístico e integrador.

Vygotsky (1986) fue uno de los pocos investigadores que en su tiempo estudiaron el juego dramático en los niños. Predijo que la capacidad de los niños menores de cinco años para participar en actividades imaginativas sería un mejor indicador de éxito académico que ninguna otra actividad, incluyendo competencias verbales y cuantitativas. La razón, creía Vygotsky, era que esa participación permitía a los niños regular su comportamiento social. Contrariamente a una actividad despreocupada, como la entendemos, concibió el juego imaginativo como una experiencia en la que los niños tendrían que restringir su comportamiento.

La cascada de investigaciones confirmatorias que siguió a estas ideas llevó directamente al programa Herramientas de la Mente. Consta de muchas partes, pero las tres relevantes para nuestra discusión son el juego de planificación, la instrucción directa para la simulación y el tipo de entorno en que tiene lugar la instrucción. Los niños que participan en el programa normalmente muestran un rendimiento entre un $30 \%$ y un cien por ciento superior a los niños del control en cualquier test de función ejecutiva al que los sometamos. Esto también significa mejores notas, pues una elevada función ejecutiva es uno de los mayores predictores del éxito académico que existen en la literatura de investigación (Medina, 2010).

Vygotsky (1986) planteaba la posibilidad de que los niños y niñas en la primera infancia son capaces de autorregularse y con ello desarrollar funciones ejecutivas a nivel intelectual y social. Otro beneficio, entonces, es la prevención de dificultades de aprendizaje que podrían desarrollar niños y niñas que han dejado de jugar.

El juego, sin duda, entrega beneficios múltiples e insustituibles ante el más sofisticado aparato tecnológico, pues obliga la mayoría de las veces al contacto con otro, la solución de problemas, el establecimiento de roles, la comunicación, el afecto, la expresión corporal, la ubicación temporo-espacial, la relación con los objetos, es decir, conduce a 
niños y niñas, ecológicamente, hacia el aprendizaje y el desarrollo socioemocional, mejor que cualquier actividad académica.

Lo anterior es una competencia necesaria en todo quehacer social y académico, especialmente carente en niños con dificultades de aprendizaje.

\section{Cerebro y aprendizaje}

El hombre percibe el mundo por medio de su aparato perceptual, en un proceso interpretativo de los fenómenos que envuelve sus sentidos y su memoria. La memoria se considera como el proceso de adquisición, formación, conservación y evocación de información, y en este sentido sólo recordamos lo que precisamente grabamos en nuestros circuitos cerebrales y en consecuencia denominamos aprendizaje. Por otro lado, la percepción sería la capacidad de asociar informaciones sensoriales a la memoria y consecuentemente a la cognición, de modo tal que es capaz de formar conceptos sobre el mundo que nos rodea, sobre nosotros mismos, $y$ en definitiva orientar nuestro comportamiento (Lent, 2001).

La neurociencia cognitiva tiene como foco de atención la comprensión de las actividades cerebrales y los procesos de cognición, enfocando sus estudios en el aprendizaje humano, que lo entiende no como un simple proceso de almacenamiento de datos perceptuales, sino más bien, como un proceso de elaboración de las informaciones que provienen de las percepción y el cerebro. El individuo se encuentra en permanente búsqueda de respuestas para sus percepciones, pensamientos y acciones, generando una constante reorganización de sus conexiones cerebrales. La actividad mental, por su parte, estimula la reconstrucción de conjuntos neuronales, procesando experiencias, vivencias y lenguaje en un flujo y reflujo constante de información.

Las informaciones, que son captadas por los sentidos y transformadas en estímulos eléctricos que recorren las neuronas, son catalogadas y archivadas en la memoria y consecuentemente esa es precisamente la capacidad de agregar datos a informaciones ya almacenadas en la memoria, estableciendo relaciones entre lo nuevo y lo ya conocido, reconstruyendo lo que ya fue aprendido, en un reprocesamiento constante de 
interpretaciones, cuestión que caracteriza la plasticidad cerebral. En este contexto, el aprendizaje sería el proceso en virtud del cual se asocian cosas u eventos del mundo, gracias a lo cual adquirimos nuevos conocimientos. Por otro lado, la memoria es el proceso por el cual conservamos esos conocimientos a lo largo del tiempo (Mora, 2004; Ratey, 2001).

En cuanto a los sistemas cognitivos, son aquellos sistemas mentales que rigen las actividades diarias del ser humano como leer, escribir, conversar, planear o reconocer rostros. Algunos sistemas comportan otros sistemas, agregando una complejidad creciente en la generación de un comportamiento. Por su parte, los diferentes sistemas cognitivos tienen como base distintas operaciones mentales. Estas operacione mentales están relacionadas con redes neuronales de áreas cerebrales específicas (Posner \& Raichle, 2001).

Considerando la flexibilidad del cerebro para regular las demandas del ambiente, cuestión que se explica por la sinaptogénesis -capacidad de formación de nuevas conexiones, sinapsis, entre las células cerebrales -, por su parte la plasticidad cerebral, modifica química, anatómica y fisiológicamente el cerebro, puesto que exige alteraciones en las redes neuronales cada vez que las situaciones vivenciadas en el ambiente inhiben o estimulan el surgimiento de nuevas sinapsis a través de la liberación de neurotransmisores. En este sentido, ofrecer situaciones de aprendizaje fundamentadas en experiencias ricas en estímulos, fomentando a la par actividades intelectuales, podría promover la activación de nuevas sinapsis. Por su parte, una nueva información podría, por el desorden que genera, llevar a la evolución del conocimiento del individuo, puesto que el cerebro requerirá desarrollar estrategias cognitivas a fin de reorganizar y retomar el equilibrio en la construcción de nuevo conocimiento. Lo anterior, por medio de un proceso dinámico y recursivo presente en la reconstrucción del propio acto de conocer.

Para Maturana y Varela (2002) y Maturana (2004) el aprendizaje surge de un acoplamiento estructural, vale decir, las interacciones recíprocas entre individuo y medio hacen surgir cambios estructurales en la organización del ser vivo y del contexto en que está inserto, generando un proceso de auto-organización que opera con propiedades emergentes, a fin de adaptarse a las condiciones cambiantes que se encuentran presentes en 
el fenómeno del conocer. En este sentido comprender el aprendizaje como acoplamiento estructural implica que las experiencias de aprender en contextos pedagógicos generan alteraciones en la estructura del individuo, de tal manera que las experiencias de aula estimulan reflexiones recursivas sobre los pensamientos, sentimientos $\mathrm{y}$ acciones, permitiendo que el aprendizaje sea concebido como proceso reconstructivo, generando autorreorganización mental y emocional de aquellos que integran el contexto pedagógico especifico.

Se plantea, también, que aprender no es solamente reconocer lo que virtualmente ya era conocido, sino más bien transformar lo desconocido en conocimiento, en un ejercicio de reconocimiento y de descubrimiento, por tanto aprender comporta la unión de lo conocido y lo desconocido. En este mismo sentido es posible afirmar que la memoria y el aprendizaje son fundamentales para la evolución del individuo como ser social, pues superan y trascienden la simple comprensión de información por parte del sujeto que aprende, pasando a fundamentar su pensamiento y su acción (Rosas y Sebastián, 2004; Morín, 1994).

Con respecto a las emociones, estas juegan un papel decisivo en el aprendizaje, toda vez que el sistema límbico, formado por el tálamo, amígdala, hipotálamo e hipocampo, evalúa las informaciones provenientes del medio, decidiendo qué estímulos deben ser considerados y cuales descartados. Es así como cuando los seres humanos conseguimos establecer una relación entre información nueva y la memoria preexistente, son liberados neurotransmisores como la acetilcolina y dopamina, que aumentan la concentración favoreciendo la sensación de satisfacción. De tal modo que la razón se encuentra

fuertemente relacionada con la emoción, puesto que nuestros actos y pensamientos son siempre influenciados por las emociones (Damasio, 2000; 2006).

\section{Juego y aprendizaje}

En la actualidad son numerosos los estudios que sugieren la existencia de fuertes vínculos entre cerebelo, la memoria, la percepción espacial, el lenguaje, la atención, la emoción, las claves no verbales e incluso la toma de decisiones, cuestión que implica con fuerza el movimiento y el juego para fomentar la cognición. 
El hecho de realizar diferentes tipos de juegos (a distintas intensidades), con compromiso motor relevante, puede generar los siguientes beneficios (Jensen, 2010):

- La zona conocida como cingulada anterior se activa especialmente cuando se realizan nuevos movimientos o combinaciones de estos, en consecuencia variar o complejizar un juego permitiría la creación de nuevos movimientos.

- La integración sensoriomotriz es fundamental en la preparación y éxito en la escuela, en este sentido las áreas como el lenguaje y las matemáticas se deberían articular y conectar con expresiones motoras diversas.

- Los estudios han demostrado que los ratones que crecieron en entornos enriquecidos, con mucho juego y movimiento, tenían un mayor número de conexiones entre las neuronas y mayor capitalización, que los que no disponían de ese tipo de entornos.

- El ejercicio y el movimiento, propios del juego, fortalece los ganglios basales, el cerebelo y el cuerpo calloso, zonas claves del cerebro, además de aportar neurotropinas (alimento alto en nutrientes) para mejorar el crecimiento y establecer más conexiones entre neuronas.

- Divertirse y jugar es bueno para la salud, disminuye el estrés y mejora el funcionamiento del sistema inmunitario.

Finalmente Jensen (2010: 124) plantea que "los niños que disfrutan con los juegos en el patio lo hacen por una buena razón: las experiencias sensoriomotrices alimentan directamente los centros del placer de sus cerebros".

Qué más interesante y desafiante que el hecho de aprender jugando. Es posible construir una pedagogía de lo lúdico, conectado eficaz y eficientemente con el aula escolar. A continuación se intentará un paralelo entre la educación predominante en nuestro tiempo y ampliamente documentada (Avalos, 2002; Calvo, 2007; Bohm y Schiefelbein, 2008) y una propuesta que surge desde la experiencia de años en el aula escolar y de la necesaria reflexión a la luz de las neurociencias: 
- En la escuela los niños deben desvincularse, separarse de sus gustos e intereses para 'poner atención' a las materias.

- El aprendizaje no se da exclusivamente en el sistema escolar formal, no es posible pedirle a los niños que no traigan su capital cultural, sus gustos, intereses e inquietudes a la escuela, puesto que esto es negar al otro como un otro legitimo (Maturana, 1999).

- En el periodo escolar se dedica el niño y la niña selectivamente a una materia y luego a otra.

- La propuesta es que la escuela sea la continuación de la vida, los niños aprenden en su casa, en la calle, en el patio, en el recreo, en la micro, en los camarines, en fin, la vida se despliega en todo momento. En consecuencia el aprendizaje no debería estar fragmentado, normalmente en el día a día no decimos ahora voy a pensar y razonar en matemáticas, o en ciencias, muy por el contrario siempre pienso desde la totalidad de los fenómenos.

- En la sala de clases debe primar el orden, el silencio, la disciplina, el 'respeto', no las risas, el juego o lo lúdico.

○ ¿Es posible aprender jugando? Desde la experiencia como educadores es posible cuando el aula se transforma en un espacio conversacional distinto, donde la espontaneidad, honestidad y el respeto priman, puesto que dramatizar una batalla, escenificar un circo, bailar, jugar a los títeres, las marionetas, danzar, rescatar los juegos tradicionales, nos permiten contextualizar y fortalecer los contenidos de aprendizaje, con un espíritu lúdico que transversalice el currículo. Así como un niño(a) juega, ríe y aprende en casa, es deseable y posible que continúe riendo, jugando y aprendiendo en la escuela.

En este contexto la investigación nos señala que la pedagogía de un educador es uno de los aspectos más importantes a la hora de evaluar la calidad del aprendizaje de los niños. Así que los educadores de la primera infancia deben considerar con cuidado y cuestionar su pedagogía y las prácticas correspondientes. En la ejecución práctica de aula, 
los educadores deben discutir y describir su comprensión de los principios de la práctica. Una de las prácticas más utilizadas en el sector de la primera infancia es el 'aprendizaje a través del juego'. El aprendizaje basado en el juego es descrito como un contexto para el aprendizaje a través del cual los niños se organizan y dan sentido a sus mundos sociales, ya que se comprometen activamente con las personas, los objetos y las representaciones (Barblett, 2010).

El juego físicamente activo permite a los niños probar y desarrollar todo tipo de habilidades motoras. Promueve los beneficios de salud y de bienestar importantes. Los centros educativos de alta calidad han incorporado un programa de aprendizaje basado en el juego, considerando los siguientes aspectos:

- Un programa diario que incluye el juego físico de interior y al aire libre.

- La integración de la música, el movimiento y la expresión creativa.

- Interacciones entre adultos y niños realizando actividad física. Esto significa que los educaores son a veces tan comprometidos físicamente en el juego activo como los niños.

La reproducción no se da en el vacío; por lo general se lleva a cabo dentro de un espacio físico y social. Uno de los mayores beneficios del juego es ayudar con el desarrollo de la competencia social. Los niños pueden construir relaciones, aprender a resolver conflictos, negociar y regular sus comportamientos. En el juego, los niños por lo general inincrementan los sentimientos de éxito y optimismo, ya que actúan como sus propios agentes y de tomar sus propias decisiones. Jugar es una liberación de estrés; a menudo está vinculado con el bienestar infantil. En sentido, concordamos con los enunciados de Rosas (2008: 203), quien relaciona las nociones de aprendizaje y juego:

- Todo juego debe entretener.

- Para que un juego sea considerado entretenido, el único criterio válido en la entretención del niño que juega, mientras lo juega.

- El juego debe diseñarse al nivel cognitivo del niño que juega. 
- Primero se debe jugar con los significados de objetos y acciones, antes que el niño comprenda el significado de dichos objetos y acciones.

- El juego debe posibilitar la construcción de un espacio imaginario donde pueda manejar libremente los objetos y conceptos que se deseen instruir.

- Es preferible el uso del juego cooperativo a los juegos aislados: la posibilidad de ampliación recíproca de Zonas de Desarrollo Próximo aumenta exponencialmente con la cantidad de participantes.

- El nivel de desarrollo de los niños que juegan cooperativamente debiera no ser demasiado diferente.

- La invitación a jugar no debe ser confundida con la invitación a trabajar.

- No es posible (ni deseable) enseñarlo todo por medio del juego. Sólo se pretende crear espacios imaginarios de ampliación de Zona de Desarrollo Próximo.

- El juego es una cosa muy seria.

\section{A modo de conclusión}

La necesidad de aproximarnos a los hallazgos del área de las neurociencias de la educación, sustenta la premisa de que las instituciones responsables de la formación de futuras educadoras requieren examinar sus planes formativos y discutir los componentes curriculares, a objeto de que las profesionales egresadas posean una comprensión más actualizada de las nociones de aprendizaje y juego, en el marco del desarrollo de los nuevos enfoques de la cognición.

Si consideramos la gama de posibilidades de aprendizaje que desarrollan los niños a través del juego. ¿Cuántos indicadores de posibles dificultades de aprendizaje podríamos detectar?; ¿podríamos integrar programas especiales de juego en los jardines infantiles a objeto de que en conjunto con las educadoras se evalúen diferentes aspectos cognitivos, sociales y emocionales que puedan brindar información sobre las fortalezas y debilidades que presentan niños y niñas en temprana edad, siendo derivados a tiempo y pertinentemente al profesional correspondiente? 
Podríamos con ello disminuir el impacto que causan las dificultades de aprendizaje en la enseñanza básica, interviniendo a través del en la primera infancia; ello con la intención de construir un aprendizaje desde la totalidad que considera las diversas dimensiones de la persona, con una mirada integradora desde lo biológico, social, cognitivo, emocional, relacional y contextual.

Desde nuestra experiencia parece total y absolutamente posible. Es más, nos imaginamos entre risas y conversaciones pequeñas, jugando a las bolitas, o a las carreras de autos, o tomando una taza de té en sillas diminutas, rodeadas de manos pequeñas y voces alegres, en una renovada aula de primera infancia, en que los principios y orientaciones de los nuevos desarrollos de la neurociencia hacen del juego una maravillosa y especial instancia de aprendizaje.

\section{Referencias bibliográficas}

Avalos, B. (2002). Profesores para chile, historia de un proyecto. Santiago de Chile: Ministerio de Educación.

Barblett, L. (2010). Why play-based learning?. Every Child Magazine. 16, 1, s/p.

Bohm, W. y Schiefelbein, E. (2008). Repensar la educación. Diez preguntas para mejorar la docencia. Santiago de Chile: Editorial Andrés Bello.

Calvo, C. (2008). Del mapa escolar al territorio educativo. Disoñando la escuela desde la educación. Santiago de Chile: Editorial Nueva Mirada.

Damasio, A. (2000). Sentir lo que sucede, cuerpo y emoción en la fábrica de la conciencia. Santiago de Chile: Andrés Bello.

Damasio, A. (2006). El error de Descartes, 4ta, Impresión. Barcelona: Crítica.

Freire, P. (2002). Pedagogía de la esperanza, un reencuentro con la pedagogía del oprimido. Buenos Aires: Siglo XXI.

Jensen, E. (2010). Cerebro y Aprendizaje, competencias e implicaciones educativas. Madrid: Narcea. 
Lent, R. (2001). Cien billones de neuronas: conceptos fundamentales de la neurociencia. Sao Paulo: Ateneo.

Maturana, H. (1999). Emociones y lenguaje en educación y política. Santiago de Chile: Lom.

Maturana, H. (2004). Desde la biología a la psicología. Santiago de Chile: Editorial Universitaria.

Maturana, H. y Varela, F. (2002). El árbol del conocimiento. Santiago de Chile: Editorial Universitaria.

Medina, J. (2013). Viaje al cerebro del niño. Barcelona: Paidós.

Mora, F. (2004). Cómo funciona el cerebro. Porto Alegre: Artmed.

Morin, E. (1994). El método III. El conocimiento del conocimiento. Madrid: Cátedra.

Morin, E. (2001). Los siete saberes necesarios para la educación del futuro. Barcelona: Paidos.

Morin, E. (2005). Introducción al pensamiento complejo. Octava reimpresión. Barcelona: Gedisa.

Posner, M. y Raichle, M. (2001). Imágenes de la mente. Porto: Porto Editora.

Ratey, J. (2001). El cerebro: una guía para el usuario. Rio de Janeiro: Objetiva.

Rosas, R. y Sebastian, C. (2004). Piaget, Vygotsky y Maturana. Constructivismo a tres voces. Buenos Aires: AIQUE.

Rosas, R. (2008). Juego, aprendizaje implícito y metacognición: hacia un modelo para el desarrollo de juego educativos. En Kronmuller, C. y Cornejo C. (2008) (Eds). Ciencias de la Mente: aproximaciones desde latinoamerica. Santiago de Chile: J. C. Saez.

UNICEF (2012). Early Learning. Recuperado el 06-08-2015 de http://www.unicef.org/earlychildhood/index_40747.html

Vigotski, L. (1986). La imaginación y el arte en la infancia. Madrid: Akal. 